\title{
Berichtigung
}

Auf Seite VII, letzte Zeile, muß es richtig heißen:

Zum Problem der Triebkräfte...

2087/16 - Kumpf-Korfes, Bismarcks ,"Draht nach Rußland“ 

SIGRID KUMPF-KORFES

BISMARCKS „DRAHT NACH RUSSLAND“ 
DEUTSCHE AKADEMIE DER WISSENSCHAFTEN ZU BERLIN INSTITUT FUR GESCHICHTE

ARBEITSG RUPPE FUR GESCHICHTE DER SLAWISCHEN VOLLKER

\title{
QUELLEN UND STUDIEN ZUR GESCHICHTE OSTEUROPAS
}

\author{
HERAUSGEGEBEN VON \\ EDUARD WINTER \\ UND \\ HEINZ LEMKE \\ IN ZUSAMMENARBEIT MIT \\ ALFRED ANDERLE, CONRAD GRAU, GUNTER ROSENFELD UND \\ FRITZ STRAUBE \\ REDAKTIONSSE KRETÅR: GUNTHER JAROSCH \\ BAND XVI
}




\section{SIGRID KUMPF-KORFES}

\section{BISMARCKS \\ „DRAHT NACH RUSSLAND“}

Zum Problem der sozial-ökonomischen Hintergründe der russisch-deutschen Entfremdung im Zeitraum von 1878 bis 1891

AKADEMIE-VERLAG - BERLIN 1968 
Erschienen im Akademie-Verlag GmbH, 108 Berlin, Leipziger Straße 3-6

Copyright 1968 by Akademie-Verlag $\mathbf{G m b H}$

Lizenznummer: $202 \cdot 100 / 37 / 68$

Herstellung: IV/2/14 VEB Werkdruck, 445 Gräfenhainichen $\cdot 2854$

Bestellnummer: $2087 / 16 \cdot$ ES 14 D 
Meinem Vater Otto Korfes 
